\title{
Citrulinemia tipo I con insuficiencia hepática recurrente en un niño
}

\author{
Citrullinemia type I with recurrent liver failure in a child
}

\author{
Dra. Verónica Bindia y Dr. Hernán Eiroa ${ }^{a}$
}

\begin{abstract}
RESUMEN
La citrulinemia tipo I es un desorden autosómico recesivo causado por la mutación del gen ASS1, que expresa argininosuccinato sintetasa, enzima limitante del ciclo dela urea. Las variantes clásicas están asociadas con la forma neonatal/ infantil, que llevan a hiperamoniemia y a la muerte si el tratamiento no es instaurado.

Los síntomas iniciales de los trastornos del ciclo de la urea incluyen deterioro neurológico con leve o moderado daño hepático.

Reportamos un caso de falla hepática recurrente en un lactante con diagnóstico de citrulinemia tipo I sin compromiso neurológico grave, que fue derivado a nuestro centro para trasplante hepático.

La falla hepática aguda puede ser causada por una gran variedad de desórdenes, dentro de los que se incluyen errores congénitos del metabolismo. El tratamiento adecuado de los trastornos del ciclo de la urea y, en particular, la citrulinemia I puede evitar la necesidad de un trasplante.

Palabras clave: citrulinemia, trastornos innatos del ciclo de la urea, falla hepática, pediatría, trasplante hepático.
\end{abstract}

\begin{abstract}
Citrullinemia type I is an autosomal recessive disorder caused by mutation of the gene expressing ASS1 argininosuccinate synthetase, limiting enzyme of the urea cycle.

The classic variants are associated with neonatal/infantile forms that cause hyperammonemia leading to death if treatment is not established.

Initial symptoms of disorders of the urea cycle include neurological impairment with mild or moderate liver damage. We report a case of recurrent liver failure in an infant diagnosed with type I citrullinemia without severe neurological involvement that was referred to our center for liver transplantation.

Acute liver failure can be caused by a wide range of disorders in which inborn errors of metabolism are included. Appropriate treatment of disorders of the urea cycle and in particular citrullinemia I can avoid the need for a transplant.

Key words: citrullinemia, inborn urea cycle disorders, pediatric, liver failure, liver transplantation.

http:/ /dx.doi.org/10.5546/aap.2017.e39
\end{abstract}

a. Servicio de Errores Congénitos del Metabolismo.

Hospital de Pediatría SAMIC "Prof. Dr. Juan P. Garrahan".

Correspondencia:

Dra. Verónica Bindi, verogbindi@gmail.com

Financiamiento: Ninguno.

Conflicto de intereses: Ninguno que declarar.

Recibido: 31-5-2016

Aceptado: 15-9-2016

\section{INTRODUCCIÓN}

La citrulinemia tipo I es un trastorno del ciclo de la urea causado por la deficiencia de argininosuccinato sintetasa, cuya función es convertir la citrulina y aspartato en argininosuccinato. ${ }^{1}$

La incidencia de citrulinemia tipo I es estimada en 1:250000 nacidos según estudios de pesquisa neonatal por espectrometría de masas., ${ }^{2,3}$

La enfermedad se presenta como una encefalopatía hiperamoniémica en el período neonatal o la infancia y lleva a la muerte o morbilidad. Otras formas de presentación son falla de medro, problemas de conducta, aversión a las proteínas o síntomas desencadenados por catabolismo. ${ }^{1}$ Se describen situaciones en el período de embarazo o posparto que se presentan con falla hepática o hígado graso agudo del embarazo. ${ }^{4}$

La función hepática durante los episodios de descompensación, usualmente, está conservada; puede hallarse un aumento de transaminasas. ${ }^{1}$ La falla hepática transitoria es reconocida como un raro signo de presentación en los trastornos del ciclo de la urea. Los pacientes previamente reportados presentaron deterioro neurológico y deterioro agudo de la función hepática, que fueron superados con el tratamiento de la enfermedad de base sin necesidad de trasplante, ${ }^{5,6}$

Reportamos un caso de falla hepática recurrente en un lactante diagnosticado y seguido en nuestro centro por 6 años con diagnóstico de citrulinemia tipo I sin compromiso neurológico grave, que fue derivado, inicialmente, para trasplante hepático.

\section{CASO CLÍNICO}

Paciente de sexo masculino, primer hijo de una pareja sana no consanguínea. Como antecedentes relevantes, había presentado retraso del crecimiento a partir del año de vida. Crecía en las curvas de peso y talla por debajo de 2 desvíos estándar (DE), con perímetro cefálico en -2 DE desde entonces. El desarrollo madurativo era acorde a la edad.

A los 18 meses, comenzó con vómitos y 
rechazo al alimento de 15 días de evolución asociados a la elevación de transaminasas en el contexto de infección de la vía aérea superior. Fue derivado al Hospital Garrahan por el empeoramiento progresivo de la función hepática para trasplante.

Al momento del examen físico, no presentaba hepatomegalia, ictericia o signos de encefalopatía. El análisis de laboratorio de ingreso revelaba transaminasa glutámico-oxalacética (glutamic oxaloacetic transaminase; GOT, por sus siglas en inglés): $814 \mathrm{UI} / \mathrm{L}$; transaminasa glutámicopirúvica (glutamic-pyruvic transaminase; GPT, por sus siglas en inglés): $1250 \mathrm{UI} / \mathrm{L}$; fosfatasa alcalina (FAL): 810; gamma-glutamil transpeptidasa (GGT): 141; albúmina: 3,2 mg/dl; tiempo de protrombina (TP): $42 \%$; tiempo de tromboplastina parcial activada con caolín (kaolin activated partial tromboplastin time; KPTT, por sus siglas en inglés): 94 segundos; relación internacional normalizada (RIN): 6,5; amoniemia: $365 \mathrm{mg} / \mathrm{dl}$; sin acidosis; normoglucémico (Figuras 1 y 2, semanas 1 y 2 ). Se solicitó, al día siguiente, una nueva determinación de amonio por no correlacionarse con la clínica y este resultado fue normal, por lo que se consideró este valor elevado previo como error preanalítico.

Las hepatitis de etiología infecciosa fueron descartadas y la alfa-1-antitripsina era normal. En la ecografía abdominal, el tamaño hepático estaba conservado.
Durante esta internación, al inicio, permaneció ayunado con un flujo de glucosa de $4 \mathrm{mg} / \mathrm{k} /$ minuto por 48 horas; luego se alimentó con hidrolizado proteico. Se observó una mejoría paulatina espontánea de la función hepática, por lo que fue derivado a su provincia.

Un mes más tarde, comenzó con la misma sintomatología de falla hepática aguda acompañada de vómitos, sin compromiso neurológico grave.

De acuerdo con el análisis de laboratorio inicial, ingresó con GOT: 848 UI/L; GPT: 1124 UI/L; TP: 26\%; KPTT: 66 segundos; amonio: $270 \mathrm{mg} / \mathrm{dl}$.

El análisis cuantitativo de aminoácidos plasmáticos reveló glutamina: $1700 \mu \mathrm{mol} / \mathrm{L}$ (420-700 $\mu \mathrm{mol} / \mathrm{L})$; citrulina: $1711 \mu \mathrm{mol} / \mathrm{L}(2-$ $30 \mu \mathrm{mol} / \mathrm{L})$; arginina: 13,3 $\mathrm{mol} / \mathrm{L}(2-50 \mu \mathrm{mol} / \mathrm{L})$; alanina: $122 \mu \mathrm{mol} / \mathrm{L}(70-345 \mu \mathrm{mol} / \mathrm{L}) ; \mathrm{y}$ presencia de ácido orótico en orina. Este perfil bioquímico fue compatible con citrulinemia I.

El diagnóstico se confirmó con el estudio molecular del gen ASS1 localizado en el cromosoma 9q34.1, que detectó la mutación heterocigota c.970 G>A (p.Gly324Ser) y c.1168G >A (p.Gly390Arg). Ambas mutaciones ya fueron descritas [7]. La segunda se reportó en Argentina asociada a una forma grave neonatal. 8,9

Con el diagnóstico de citrulinemia I, comenzó el tratamiento con flujo de glucosa, arginina

FIGURA 1. Evolución de los resultados de los valores de transaminasas y amonio a lo largo del tiempo

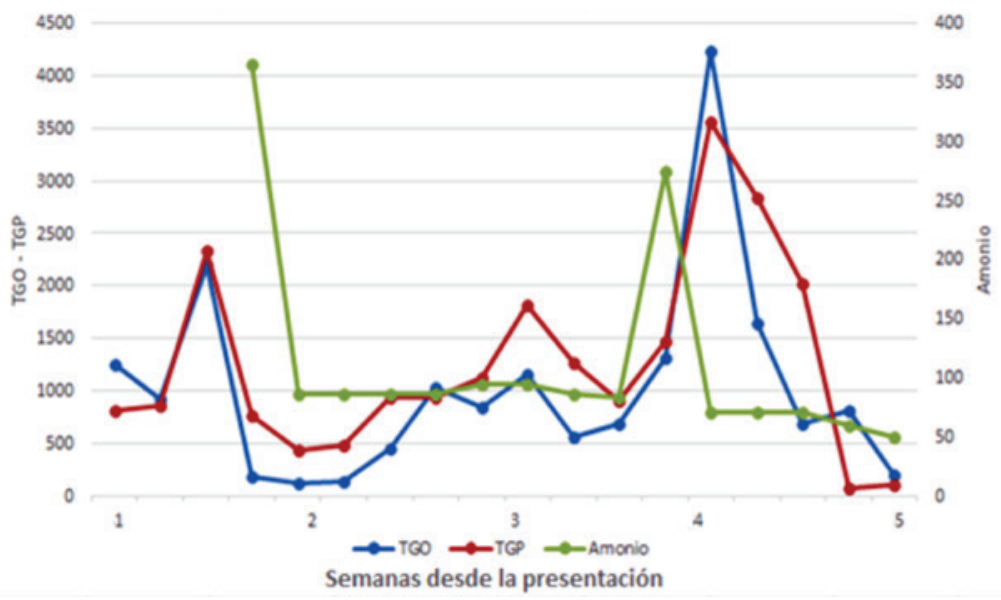

TGO: transaminasa glutámico-oxalacética; TGP: transaminasa glutámico-pirúvica.

Transaminasas (valores normales -VN-: 30-40 UI/L) y niveles de amonio (VN: hasta $120 \mathrm{mg} / \mathrm{dl}$ ) durante el trascurso de las semanas desde la falla hepática. En las semanas 1 y 2, hay un aumento de los tres valores con mejoría posterior parcial en las semanas 2 y 3, con aumento de amonio previo a las transaminasas en el segundo pico. Nótese la mejoría de los parámetros con el tratamiento (semana 5). 
(300 mg/k/día) y benzoato de sodio (250 mg/k/ día). Los parámetros clínicos y bioquímicos de la función hepática mejoraron en 48 horas. El paciente recibió, a partir del segundo día del diagnóstico, dieta con un aporte proteico restringido y calórico adecuado.

Los máximos niveles alcanzados por las enzimas hepáticas (Figura 1) fueron días previos al diagnóstico, que coincidió con el detrimento de la función hepática, medido a través de TP y RIN (Figura 2).

La mejoría inicial se debió al ayuno por la intolerancia oral y flujo de glucosa instaurado.

El tratamiento específico de la enfermedad comenzó en la semana 5 (S5), que coincidió con la mejoría de los parámetros de la función hepática y el descenso del amonio (Figuras 1 y 2).

Actualmente, tiene 6 años y su dieta se basa en 1,2 g/kilo de proteínas con un 0,2 g/ kilo de aminoácidos esenciales, que le aportan $70 \mathrm{kcal} / \mathrm{k} /$ día, y formulaciones orales de arginina y benzoato de sodio. Desde entonces, no ha tenido graves descompensaciones. El peso y la talla se encuentran en el percentilo 75 . Su desarrollo neurocognitivo y motor es normal, según la apreciación del pediatra. El Servicio de Psicopedagogía realizó un perfil cognitivo mediante la escala de inteligencia de Wechsler para preescolar y primaria IV (Wechsler Preschool and Primary Scale of Intelligence IV; WPPSI IV, por sus siglas en inglés), que dio un perfil discrepante entre comprensión verbal (cociente intelectual
-CI-: 75) y visoespacial (CI: 98). Esto indicó un menor desarrollo verbal con respecto a la esfera visoespacial, pero se encontró aún en los percentilos esperados para la edad.

No ha presentado daño neurológico ni hepático en el seguimiento longitudinal. No ha sido evaluado por un neurólogo infantil.

Tiene una hermana de un año que padece la misma enfermedad, diagnosticada en estado presintomático dado el antecedente.

\section{DISCUSIÓN}

La falla hepática aguda puede ser definida según los siguientes criterios: evidencia bioquímica de lesión hepática; sin compromiso hepático previo; coagulopatía que no se corrige con vitamina K; RIN menor de $1,5{ }^{10}$

La presentación de los trastornos del ciclo de la urea, como disfunción hepática, no es frecuente. Se describen, en la literatura, otros trastornos del ciclo de la urea, como síndrome de hiperornitinemia, hiperamoniemia y homocitrulinemia y déficit de ornitina transcarbamilasa, que también pueden presentarse como disfunción hepática. En todos los casos, el compromiso neurológico estaba presente.

En la literatura, se describen pocos casos de esta forma de presentación de citrulinemia I, todos con cuadro de encefalopatía hiperamoniémica previa. ${ }^{5,6}$

El compromiso neurológico en un lactante

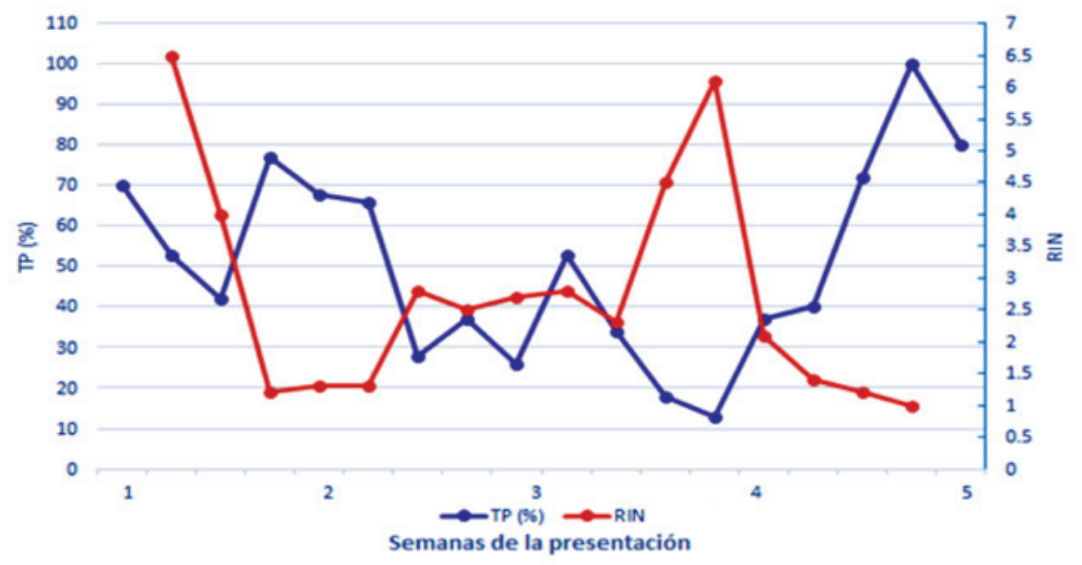

TP: tiempo de protrombina; RIN: relación internacional normalizada.

Valores de TP y RIN (parámetros de función hepática). Valores normales: TP de 70\%-100\% y RIN de 0,9 a 1,2. El ascenso del RIN es inversamente proporcional al descenso del TP. Mejoran ambos parámetros con el tratamiento durante las últimas semanas. 
puede ser sutil y difícil de evaluar. En este reporte, el paciente no presentó clínica neurológica grave; sí tuvo daño hepático y retraso del crecimiento con rechazo al alimento, como se describe en la literatura. ${ }^{1}$

El pico máximo alcanzado de amonio fue de $270 \mathrm{mg} / \mathrm{dl}$. Este valor no generó daño neurológico evidente frente a la evaluación del pediatra. No fue evaluado por un neurólogo en este caso, por lo que consideramos que es una limitación de este reporte.

La fisiopatología de la disfunción hepática no se conoce del todo. Existe evidencia de que los intermediarios del ciclo de la urea causan disfunción mitocondrial en el hígado. ${ }^{11}$ Esto correlaciona en la literatura y en este reporte, en que el pico de amonio precede al empeoramiento de la función hepática, por lo que podría relacionarse con tóxicos hepáticos circulantes. ${ }^{12}$

Sin embargo, la disfunción es independiente del defecto enzimático, metabolitos intermediarios o número de episodios de hiperamoniemia y puede ocurrir con o sin ella. ${ }^{11}$

Los trastornos del ciclo de la urea se pueden diagnosticar por pesquisa neonatal ampliada, lo que implicaría disminuir las demoras en el diagnóstico e instaurar una adecuada terapéutica. ${ }^{2,3}$

El tratamiento etiológico de la enfermedad logró diferir el trasplante hepático en este caso, por lo que se resalta la importancia de definir la etiología de la falla.

\section{REFERENCIAS}

1. Brusilow SW, Horwich AL. Urea cycle enzymes. En: Scriver C, Beaudet A, Sly W, Valle D, et al. The Metabolic and Molecular Bases of Inherited Disease. $8^{\text {th }}$ ed. New York: McGraw-Hill, 2001.Págs.1909.

2. Kasper DC, Ratschmann R, Metz TF, Mechtler TP, et al. The national Austrian newborn screening program - eight years experience with mass spectrometry. Past, present, and future goals. Wien Klin Wochenschr 2010;122(21-22):607-13.

3. Summar ML, Koelker S, Freedenberg D, Le Mons C, et al. The incidence of urea cycle disorders. Mol Genet Metab 2013;110(1-2):179-80.

4. Ito S, Kurasawa G, Yamamoto K, Furuta I, et al. A pregnant patient with fulminant hepatic failure was found to carry a novel missense mutation in the argininosuccinate synthetase gene. J Gastroenterol 2004;39(11):1115-7.

5. Faghfoury H, Baruteau J, de Baulny HO, Häberle J, et al. Transient fulminant liver failure as an initial presentation in citrullinemia type I. Mol Genet Metab 2011;102(4):413-7.

6. De Groot MJ, Cuppen M, Eling M, Verheijen F, et al. Metabolic investigations prevent liver transplantation in two young children with citrullinemia type I. J Inherit Metab Dis 2010;33(Suppl 3):S413-6.

7. Berning C, Bieger I, PauliS, Vermeulen T, etal. Investigation of citrullinemia type Ivariants by in vitro expression studies. Hum Mutat 2008;29(10):1222-7.

8. Larovere LE, Ruiz SM, Angaroni CJ, de Kremer RD. Molecular epidemiology of citrullinemia type I in a risk region of Argentina: a first step to preconception heterozygote detection. JIMD Rep 2012;6:27-9.

9. Larovere LE, Angaroni CJ, Antonozzi SL, Bezard MB, et al. Citrullinemia type I, classical variant. Identification of ASS-p G390R (c.1168G >A) mutation in families of a limited geographic area of Argentina: a possible population cluster. Clin Biochem 2009;42(10-11):1166-8.

10. Ciocca M. Insuficiencia hepática. PRONAP 10. Módulo 1. Buenos Aires: SAP; 2010.Págs.8-29.

11. Zamora S, Pinto A, Scott R, Parsons H, Mitochondrial abnormalities of liver in two children with citrullinaemia. J Inherit Metab Dis 1997;20(4):509-16.

12. Lücke T, Tsikas D, Kanzelmeyer N, Vaske B, et al. Elevated plasma concentrations of the endogenous nitric oxide synthase inhibitor asymmetric dimethylarginine in citrullinemia. Metabolism 2006;55(12):1599-603. 